\title{
On the exchange of energy in coupled Klein-Gordon equations
}

\author{
K.R. Khusnutdinova ${ }^{\mathrm{a}, \mathrm{b}, *}$, D.E. Pelinovsky ${ }^{\mathrm{c}}$ \\ a Department of Mathematical Sciences, Loughborough University, Loughborough LE11 3TU, UK \\ ${ }^{\mathrm{b}}$ Institute of Mechanics, Ufa Branch of the Russian Academy of Sciences, Karl Marx Str. 6, Ufa 450000, Russia \\ ${ }^{c}$ Department of Mathematics, McMaster University, 1280 Main Street West, Hamilton, Ont., Canada L8S 4K1
}

Received 6 August 2002; received in revised form 18 October 2002; accepted 26 November 2002

\begin{abstract}
We consider a system of coupled Klein-Gordon equations, which models one-dimensional nonlinear wave processes in two-component media. We find both linear and nonlinear solutions involving the exchange of energy between the different components of the system. The solutions are a continuum generalization of the classical example of energy exchange in Mandelshtam's system of coupled pendulums.

() 2003 Elsevier Science B.V. All rights reserved.
\end{abstract}

\section{Introduction}

We consider the system of coupled Klein-Gordon equations in the form:

$$
u_{t t}-u_{x x}=f_{u}(u, w), \quad w_{t t}-c^{2} w_{x x}=f_{w}(u, w),
$$

where subscripts denote derivatives, $f(u, w)$ is a potential function of nonlinear coupling, and $c$ the ratio of the acoustic velocities of the components $u$ and $w$. The dimensionless system (1) describes the long-wave dynamics of two coupled one-dimensional periodic chains of particles [1]. The elements of each chain are linked by a linear coupling, while the chains interact via a nonlinear coupling.

The choice of the potential function $f(u, w)$ depends on the mathematical model of the physical system. For instance, the coupled Klein-Gordon equations (1) with the proper choice of the function $f(u, w)$ can be considered as the long-wave limit of a lattice model for one-dimensional nonlinear wave processes in a bi-layer [1a]. Similar lattice models were proposed for the modeling of crack propagation in composites (see, for example, [2]). Many advanced structures, such as joints, coatings and electronic packages, use bi-materials. When applied to the modeling of bi-materials, the potential function $f(u, w)$ and parameter $c$ are determined by the type of interface and materials forming the bi-layer. In addition, we note that similar models were proposed to describe dynamical processes in hydrogen-bonded chains (e.g., [3-6]), molecular crystals and polymer chains as well as ferroelectrics or ferromagnets and thin films where rotational and vibrational degrees of freedom are coupled together (e.g., [7-12]), and in the DNA double helix [13] (see also [14] and references therein).

\footnotetext{
* Corresponding author. Present address: Department of Mathematical Sciences, Loughborough University, Loughborough LE11 3 TU, UK. Tel.: +44-1509-228202; fax: +44-1509-223969.

E-mail address: k.khusnutdinova@lboro.ac.uk (K.R. Khusnutdinova).
} 
As the function $f(u, w)$ in such phenomenological model should be found experimentally, its analytic form is not unique. Some suitable choices of $f(u, w)$ and $c$ can be found to simplify the analysis. The Lie group classification of the system (1) was carried out in [1] for $c \neq 1, f_{u w}(u, w) \neq 0$. The general system (1) with $c=1$ (and arbitrary functions of $u$ and $w$ on the right-hand side) was studied in [15], where the cases admitting Lie-Bäcklund symmetries were found, and integrable (completely or partially) examples were presented. If $f_{u w}(u, w)=0$, the system (1) splits into two independent Klein-Gordon equations, whose group classification with respect to point symmetries was given in [16] (see also [17]) and that with respect to the Lie-Bäcklund symmetries in [18].

The general system (1) is Lagrangian with the density:

$$
L=\frac{1}{2}\left(u_{t}^{2}+w_{t}^{2}-u_{x}^{2}-c^{2} w_{x}^{2}\right)+f(u, w)
$$

The system exhibits two balance equations for the conserved energy and momentum:

$$
\begin{aligned}
& \frac{\partial}{\partial t}\left[\frac{1}{2}\left(u_{t}^{2}+w_{t}^{2}+u_{x}^{2}+c^{2} w_{x}^{2}\right)-f(u, w)\right]-\frac{\partial}{\partial x}\left[u_{t} u_{x}+c^{2} w_{t} w_{x}\right]=0, \\
& \frac{\partial}{\partial t}\left[u_{t} u_{x}+w_{t} w_{x}\right]-\frac{\partial}{\partial x}\left[\frac{1}{2}\left(u_{t}^{2}+w_{t}^{2}+u_{x}^{2}+c^{2} w_{x}^{2}\right)+f(u, w)\right]=0 .
\end{aligned}
$$

When the potential function is $f(u, w)=\cos (\delta u-w)-1$, the system (1) reduces to coupled sine-Gordon equations:

$$
u_{t t}-u_{x x}=-\delta^{2} \sin (u-w), \quad w_{t t}-c^{2} w_{x x}=\sin (u-w),
$$

where the variable $u$ replaces $\delta u$, compared to the system (1). The coupled sine-Gordon equations generalize the Frenkel-Kontorova [19] dislocation model (see also [20] and references therein). Unlike the Frenkel-Kontorova model, where the shear of one part of a crystal is considered with respect to the rigid base, the system (2) is derived under the assumption that both parts of a crystal are deformable. In the model (2), the dimensionless parameter $\delta^{2}$ is equal to the ratio of masses of particles in the "lower" and the "upper" parts of the crystal. For $\delta^{2} \rightarrow 0$ and $u=0$, the system (2) reduces to the sine-Gordon equation for the variable $w$. Thus, there is a natural limit to the Frenkel-Kontorova model. We also notice that system (2) with $c=1$ was proposed to describe the open states in DNA [13].

Invariant solutions of the system (2) and solutions describing dynamics of the system in the presence of additional shear forces were constructed in [1]. It was shown that a gap appears in the spectrum of velocities of solitary waves of the system (2) if the acoustic velocities of non-interacting chains are different $\left(c^{2} \neq 1\right)$. Therefore, the system represents a filter of solitary waves (analogously to the acoustic filter in the linear theory). It was also shown that the relative displacement of the "upper" particles with respect to the "lower" particles remains the same as in the Frenkel-Kontorova model (by the period of the chain), whereas the absolute displacements depend on the wave speed.

In this paper we study in detail the linear and the nonlinear wave processes involving the exchange of energy between the two physical components of the system. In particular, we describe the full and the partial transfer of energy between the components $u$ and $w$ in the situation when one component is initially excited (say $u$ ), while the other component $(w)$ is initially at rest. The periodic and quasi-periodic processes in the wave system (2) are analogous to the energy exchange in a system of coupled pendulums in classical mechanics [21] (see also [22]).

The paper is organized as follows. In Section 2, we construct linear solutions for exchanges of energy between the two components. The exact nonlinear solution is found in Section 3 for the case $c=1$. Weakly nonlinear solutions for the general case are derived in Section 4 by means of reduction of the system (2) to coupled nonlinear Schrödinger (NLS) equations. Section 5 concludes the paper. 


\section{Energy exchange in the linear approximation}

In the case of small relative displacements of components, when $|u-w| \ll 1$, the coupled sine-Gordon equations (2) reduce to the linear system:

$$
u_{t t}-u_{x x}=-\delta^{2}(u-w), \quad w_{t t}-c^{2} w_{x x}=u-w .
$$

Fourier solutions of the system (3) take the form:

$$
u=U \mathrm{e}^{\mathrm{i}(k x-\omega t)}, \quad w=W \mathrm{e}^{\mathrm{i}(k x-\omega t)},
$$

where $\omega=\omega(k)$ satisfies the dispersion relation:

$$
D\left(\omega^{2}, k^{2}\right)=\left(\omega^{2}-\left(\delta^{2}+k^{2}\right)\right)\left(\omega^{2}-\left(1+c^{2} k^{2}\right)\right)-\delta^{2}=0
$$

and the ratio between the two components is

$$
\alpha \equiv \frac{W}{U}=\frac{-\omega^{2}+k^{2}+\delta^{2}}{\delta^{2}}=\frac{1}{-\omega^{2}+c^{2} k^{2}+1} .
$$

Due to the symmetry $k \rightarrow-k$ and $\omega \rightarrow-\omega$, we consider the dispersion curve in the first quadrant: $k>0, \omega>0$. The dispersion curve consists of two branches $\omega_{1}(k)$ and $\omega_{2}(k)$, such that $\omega_{1}(0)=0$ and $\omega_{2}(0)=\sqrt{1+\delta^{2}}$. The typical shape of the dispersion curve is shown in Fig. 1 for $c=1$ (a) and $c \neq 1$ (b). For $c=1, \omega_{1}(k)=k$ and $\omega_{2}(k)=\sqrt{1+\delta^{2}+k^{2}}$. For $c<1$ and $\delta=0$, the two branches of the dispersion curve intersect at finite $k$. The coupling $\delta \neq 0$ induces splitting of the two branches.

It is convenient to parameterize the dispersion curves $\omega=\omega_{1,2}(k)$ as follows:

$$
\omega_{1,2}^{2}=\frac{1}{2}\left[v_{1}^{2}+v_{2}^{2} \mp \sqrt{\left(v_{1}^{2}-v_{2}^{2}\right)^{2}+4 \delta^{2}}\right],
$$

where $v_{1}^{2}=\delta^{2}+k^{2}$ and $v_{2}^{2}=1+c^{2} k^{2}$, such that $\alpha_{1,2}=\left(v_{1}^{2}-\omega_{1,2}^{2}\right) / \delta^{2}=1 /\left(v_{2}^{2}-\omega_{1,2}^{2}\right)$. We notice that $\alpha_{1}>0$ and $\alpha_{2}<0$. For example, if $c=1$, then $\alpha_{1}=1$ and $\alpha_{2}=-\delta^{-2}$.

There is a pronounced energy exchange between the two components $u$ and $w$ of the system (2) when two different branches of the dispersion curve coexist for the same wavenumber $k$. Let us consider a general superposition of two linear waves with the same wavenumber $k$ but different frequencies $\omega_{1}(k)$ and $\omega_{2}(k)$ :

$$
u=U_{1} \mathrm{e}^{\mathrm{i}\left(k x-\omega_{1} t\right)}+U_{2} \mathrm{e}^{\mathrm{i}\left(k x-\omega_{2} t\right)}+\text { c.c., } \quad w=\alpha_{1} U_{1} \mathrm{e}^{\mathrm{i}\left(k x-\omega_{1} t\right)}+\alpha_{2} U_{2} \mathrm{e}^{\mathrm{i}\left(k x-\omega_{2} t\right)}+\text { c.c. },
$$

where $k>0$ and $\omega_{1,2}(k)>0$. Choosing $U_{1}$ and $U_{2}$ in such a way that the solution (7) satisfies the initial condition

$$
u(0, x)=A \cos k x, \quad w(0, x)=0,
$$

we find

$$
u=\frac{A}{\alpha_{1}+\left|\alpha_{2}\right|}\left[\left|\alpha_{2}\right| \cos \left(k x-\omega_{1} t\right)+\alpha_{1} \cos \left(k x-\omega_{2} t\right)\right], \quad w=-\frac{2 \alpha_{1}\left|\alpha_{2}\right| A}{\alpha_{1}+\left|\alpha_{2}\right|} \sin \gamma_{-} t \sin \left(k x-\gamma_{+} t\right),
$$

where $\gamma_{ \pm}=\left(\omega_{2} \pm \omega_{1}\right) / 2$.
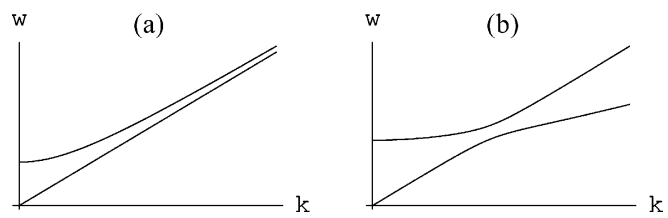

Fig. 1. The dispersion curve for $c=1$ (a) and $c \neq 1$ (b). 

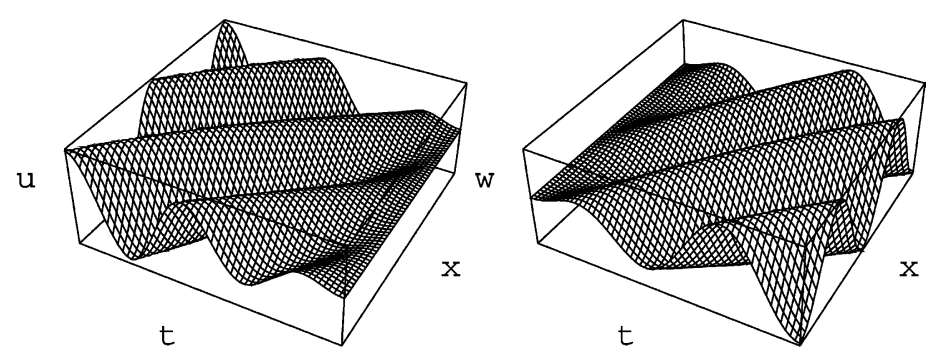

Fig. 2. Energy exchange in the linear two-wave solution (9).

A full exchange between the two components $u$ and $w$ from zero to non-zero values occurs when $v_{1}^{2}=v_{2}^{2}=v^{2}$, and therefore $\alpha_{1}=-\alpha_{2}=\delta^{-1}$. The solution (8) in this case reduces to the form

$$
u=A \cos \gamma_{-} t \cos \left(k x-\gamma_{+} t\right), \quad w=-A \delta^{-1} \sin \gamma_{-} t \sin \left(k x-\gamma_{+} t\right) .
$$

Solution (9) describes periodic exchange of energy between the two components of the coupled system with the period $T=2 \pi / \gamma_{-}$. Here, the waves propagate with the velocity $\gamma_{+} / k$, with the phases shifted by $\pi / 2$ and the amplitudes varying from 0 to the maximal value with the frequency $\gamma_{-}$. The $u$ component is at maximum at $t=0$ and at $t=\pi / \gamma_{-}$, while the $w$ component is at maximum at $t=\pi /\left(2 \gamma_{-}\right)$and so on. Thus, there is a periodic energy exchange between the $u$ and the $w$ components. Solution (9) is shown in Fig. 2 for $c=1, \delta=1, A=1, k=1.6$ in the quarter-period interval $0 \leq t \leq \pi /\left(2 \gamma_{-}\right)$.

Partial exchange between the two components $u$ and $w$ occurs when $v_{1}^{2} \neq v_{2}^{2}$. In this case, the solution (8) takes a more complicated form:

$$
u=U(t) \cos \left[k x-\omega_{2} t+\chi(t)\right], \quad w=-\frac{2 \alpha_{1}\left|\alpha_{2}\right| A}{\alpha_{1}+\left|\alpha_{2}\right|} \sin \gamma_{-} t \sin \left(k x-\gamma_{+} t\right),
$$

where

$$
U(t)=\frac{A}{\alpha_{1}+\left|\alpha_{2}\right|} \sqrt{\alpha_{1}^{2}+\alpha_{2}^{2}+2 \alpha_{1}\left|\alpha_{2}\right| \cos \left(\omega_{2}-\omega_{1}\right) t}, \quad \chi(t)=\arctan \left[\frac{\left|\alpha_{2}\right| \sin \left(\omega_{2}-\omega_{1}\right) t}{\alpha_{1}+\left|\alpha_{2}\right| \cos \left(\omega_{2}-\omega_{1}\right) t}\right] .
$$

It is clear from (10) that the amplitude of the wave propagating in the "upper" component varies between non-zero values: $u=\left(A\left|\alpha_{1}-\right| \alpha_{2}||\right) /\left(\alpha_{1}+\left|\alpha_{2}\right|\right) \cos k x$ and $u=A \cos k x$.

Solutions (9) and (10) describe the exchange of energy between the right-propagating waves. Using the symmetry $t \rightarrow-t$, one can obtain solutions for energy exchange between the left-propagating waves.

Combining all four possible waves with the same wavenumber $k$, we construct a general linear superposition for energy exchange between the two pairs of counter-propagating waves:

$$
\begin{aligned}
& u=U_{1} \mathrm{e}^{\mathrm{i}\left(k x-\omega_{1} t\right)}+U_{3} \mathrm{e}^{\mathrm{i}\left(k x+\omega_{1} t\right)}+U_{2} \mathrm{e}^{\mathrm{i}\left(k x-\omega_{2} t\right)}+U_{4} \mathrm{e}^{\mathrm{i}\left(k x+\omega_{2} t\right)}+\text { c.c. }, \\
& w=\alpha_{1}\left(U_{1} \mathrm{e}^{\mathrm{i}\left(k x-\omega_{1} t\right)}+U_{3} \mathrm{e}^{\mathrm{i}\left(k x+\omega_{1} t\right)}\right)+\alpha_{2}\left(U_{2} \mathrm{e}^{\mathrm{i}\left(k x-\omega_{2} t\right)}+U_{4} \mathrm{e}^{\mathrm{i}\left(k x+\omega_{2} t\right)}\right)+\text { c.c. }
\end{aligned}
$$

We choose the constants $U_{1,2,3,4}$ to satisfy the initial data:

$$
u(0, x)=A \cos k x, \quad w(0, x)=0, \quad \dot{u}(0, x)=\dot{w}(0, x)=0
$$

and transform the solution (11) to the form:

$$
u=\frac{A}{\alpha_{1}+\left|\alpha_{2}\right|}\left(\left|\alpha_{2}\right| \cos \omega_{1} t+\alpha_{1} \cos \omega_{2} t\right) \cos k x, \quad w=\frac{2 \alpha_{1}\left|\alpha_{2}\right| A}{\alpha_{1}+\left|\alpha_{2}\right|} \sin \gamma_{-} t \sin \gamma_{+} t \cos k x,
$$

where notations are the same as above. 

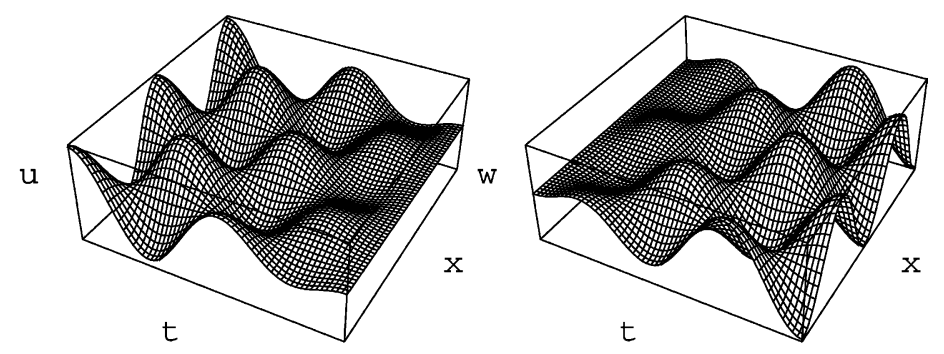

Fig. 3. Energy exchange in the linear four-wave solution (13).

If $v_{1}^{2}=v_{2}^{2}=v^{2}$, then the solution (12) takes a simple form:

$$
u=A \cos \gamma_{-} t \cos \gamma_{+} t \cos k x, \quad w=A \delta^{-1} \sin \gamma_{-} t \sin \gamma_{+} t \cos k x .
$$

Since $\gamma_{ \pm}=\left(\omega_{2} \pm \omega_{1}\right) / 2$, then $\gamma_{-}<\gamma_{+}$. The linear solution (13) describes a periodic exchange of energy with the larger period $T=2 \pi / \gamma_{-}$between the components $u$ and $w$ which represent the standing wave solutions. The process looks similar to the energy exchange in the two-wave case. It is shown in Fig. 3 for $c=1, \delta=1, A=1$, $k=1.6$ in the quarter-period interval. The amplitude of oscillations is modulated by the function $\cos k x$. For fixed $x$ we observe the classical pattern of beating between the components, shown in Fig. 4 for the point $x=0$. The energy exchange in the four-wave solution (13) is the continuum generalization of the classical example of beating oscillations in a system of coupled pendulums [21] (see also [22]).

If $v_{1}^{2} \neq v_{2}^{2}$, then

$$
u=U(t) \cos \left[\omega_{2} t-\chi(t)\right] \cos k x
$$

and the "upper" component oscillates between non-zero values $u=\left(A\left|\alpha_{1}-\right| \alpha_{2}||\right) /\left(\alpha_{1}+\left|\alpha_{2}\right|\right) \cos k x$ and $u=A \cos k x$.

If $\gamma_{-}$is small enough, i.e., if the frequencies $\omega_{1}$ and $\omega_{2}$ are close for some wave number $k$, then the $u$ component in the solutions (9) and (13) at $t=\pi / 2 \gamma_{-}$is almost in equilibrium, and its total energy is transferred to the $w$-component. At $t=\pi / \gamma_{-}$it is vice versa and so on. (Notice that physical variables in the Lagrangian formulation of the problem are $u / \delta$ and $w$.) If $v^{2} \gg \delta$, then the period $T$ of the energy exchange tends to infinity.

Let us now consider, when the condition $v_{1}^{2}=v_{2}^{2}$ is met. This condition corresponds to the full exchange (up to zero) between the two components $u$ and $w$. If $c=1$, this condition can be satisfied only for $\delta=1$, while wavenumber $k$ is arbitrary. If $c \neq 1$, then the condition $v_{1}^{2}=v_{2}^{2}$ cannot be satisfied with any wavenumber $k$, if $\left(1-\delta^{2}\right) /\left(1-c^{2}\right)<0$. Otherwise, i.e., when $\left(1-\delta^{2}\right) /\left(1-c^{2}\right) \geq 0$, the condition $v_{1}^{2}=v_{2}^{2}$ is satisfied for a single value of wavenumber $k=\left|1-\delta^{2}\right|^{1 / 2}\left|1-c^{2}\right|^{-1 / 2}$.
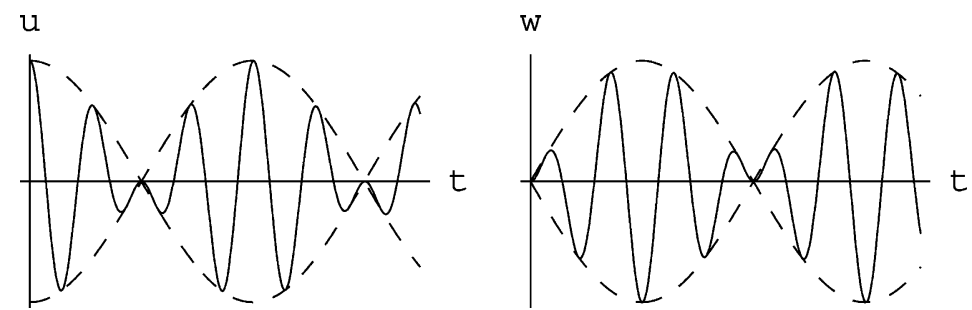

Fig. 4. Beating between the components $u$ and $w$ in the solution (13) for $x=0$. 


\section{Exact solutions for energy exchange at $c=1$}

Exact solution describing the energy exchange between the two components $u$ and $w$ of the system (2) can be constructed if $c=1$, i.e., if the acoustic velocities of the components coincide. In this case, we introduce new variables

$$
p=u-w, \quad q=u+\delta^{2} w
$$

and transform the system (2) to the form

$$
\begin{aligned}
& p_{t t}-p_{x x}=-\left(1+\delta^{2}\right) \sin p, \\
& q_{t t}-q_{x x}=0 .
\end{aligned}
$$

The system decomposes into the sine-Gordon equation (15) uncoupled from the wave equation (16). The exact periodic solution of the sine-Gordon equation (15) is (e.g., [23])

$$
p=2 \arcsin \left[\kappa \operatorname{sn}\left(k x-\omega_{2}(k) t+\theta_{0}, \kappa\right)\right],
$$

where $\omega_{2}(k)=\sqrt{1+\delta^{2}+k^{2}}$ is the second branch of the dispersion curve, $\kappa$ the modulus $(0<\kappa<1)$, and $\theta_{0}$ a constant. The general solution of the wave equation (16) has the form

$$
q=f\left(k x-\omega_{1} t\right)+g\left(k x+\omega_{1} t\right),
$$

where $\omega_{1}(k)=k$ is the first branch of the dispersion curve. We choose $g=0$ and $f$ to have the same expression as (17). As a result, the explicit two-wave solution describing the exchange of energy between the two components $u$ and $w$ can be found in terms of Jacobi elliptic functions:

$$
\begin{aligned}
& u=\frac{2}{1+\delta^{2}}\left[\arcsin \phi_{1}+\delta^{2} \arcsin \phi_{2}\right], \\
& w=\frac{2}{1+\delta^{2}}\left[\arcsin \phi_{1}-\arcsin \phi_{2}\right], \quad \phi_{1,2}=\kappa \operatorname{sn}\left(k x-\omega_{1,2} t+\theta_{0}, \kappa\right) .
\end{aligned}
$$

The solution (18) approaches the linear solution (8) as $\kappa \rightarrow 0$. When $\delta^{2}=1$ (i.e., $v_{1}^{2}=v_{2}^{2}$ ), we prove that the nonlinear solution (18) describes a full periodic exchange of energy between the two components of the system. Indeed, in this case, using the addition theorems for elliptic functions (see, e.g., [24]), we transform (18) to the form

$$
u=\arcsin \left[\frac{2 \kappa \operatorname{cn}\left(\gamma_{-} t, \kappa\right) F_{1}(t, x)}{\Phi(t, x)}\right], \quad w=\arcsin \left[\frac{2 \kappa \operatorname{sn}(\gamma-t, \kappa) F_{2}(t, x)}{\Phi(t, x)}\right],
$$

where $0<\kappa<1$ and

$$
\begin{aligned}
& F_{1}(t, x)=\operatorname{dn}\left(k x-\gamma_{+} t+\theta_{0}, \kappa\right) \operatorname{sn}\left(k x-\gamma_{+} t+\theta_{0}, \kappa\right), \quad F_{2}(t, x)=\operatorname{dn}\left(\gamma_{-} t, \kappa\right) \operatorname{cn}\left(k x-\gamma_{+} t+\theta_{0}, \kappa\right), \\
& \Phi(t, x)=1-\kappa^{2} \operatorname{sn}^{2}\left(\gamma_{-} t, \kappa\right) \operatorname{sn}^{2}\left(k x-\gamma_{+} t+\theta_{0}, \kappa\right) .
\end{aligned}
$$

Since the Jacobi function $\operatorname{dn}(z, \kappa)$ has no zeros on the real axis, zeros of the nonlinear solution (19) coincide with zeros of the functions $\operatorname{cn}(z, \kappa)$ and $\operatorname{sn}(z, \kappa)$. Zeros of the function $\operatorname{sn}(z, \kappa)$ on the real axis are located at the points $z=2 m K$, while those of the function $\operatorname{cn}(z, \kappa)$ are located at the points $z=(2 n-1) K$, where $m, n \in Z$, and

$$
K=\int_{0}^{\pi / 2}\left(1-\kappa^{2} \sin ^{2} \phi\right)^{-1 / 2} \mathrm{~d} \phi
$$

is the complete elliptic integral of the first kind. The real period of these functions is equal to $4 K$. The time of exchange of energy from one component to another is $T=K / \gamma_{-}$. The nonlinear two-wave solution (19) is shown in Fig. 5 for $c=1, \delta=1, k=1.6$, and $\kappa^{2}=0.99999$. The qualitative behaviour of the nonlinear system (2) is similar to that described in the linear approximation (see Figs. 2 and 5). 

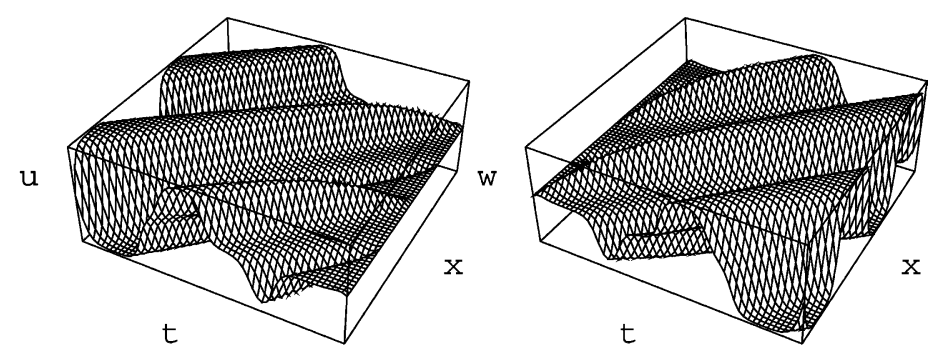

Fig. 5. Energy exchange in the nonlinear two-wave solution (19).

\section{Weakly nonlinear solution for energy exchange}

The exact solution of the system (2) for periodic exchange of energy is found only for $c=1$. This condition is very restrictive. In the general case, we can construct, however, approximate, weakly nonlinear solutions describing the energy exchange between the components $u$ and $w$. Weakly nonlinear solutions are found with the use of asymptotic methods [25] by reduction of the coupled sine-Gordon equations (2) to the coupled NLS equations. The coupled NLS equations are derived for the slow spatio-temporal evolution of the wave amplitudes. The slow evolution is captured by the asymptotic multi-scale expansion:

$$
\left(\begin{array}{l}
u \\
w
\end{array}\right)=\varepsilon\left(\begin{array}{l}
u_{1} \\
w_{1}
\end{array}\right)+\varepsilon^{2}\left(\begin{array}{l}
u_{2} \\
w_{2}
\end{array}\right)+\varepsilon^{3}\left(\begin{array}{l}
u_{3} \\
w_{3}
\end{array}\right)+\mathrm{O}\left(\varepsilon^{4}\right),
$$

where

$$
\begin{aligned}
\left(\begin{array}{c}
u_{1} \\
w_{1}
\end{array}\right)= & {\left[A(\tau, \xi) \mathrm{e}^{\mathrm{i}\left(k x-\omega_{1} t\right)}+B(\tau, \xi) \mathrm{e}^{\mathrm{i}\left(k x+\omega_{1} t\right)}\right]\left(\begin{array}{c}
1 \\
\alpha_{1}
\end{array}\right) } \\
& +\left[C(\tau, \xi) \mathrm{e}^{\mathrm{i}\left(k x-\omega_{2} t\right)}+D(\tau, \xi) \mathrm{e}^{\mathrm{i}\left(k x+\omega_{2} t\right)}\right]\left(\begin{array}{c}
1 \\
\alpha_{2}
\end{array}\right)+\text { c.c. }
\end{aligned}
$$

$\tau=\varepsilon t, \xi=\varepsilon x, T=\varepsilon^{2} t, X=\varepsilon^{2} x$ are the slow variables, and $\varepsilon$ a small amplitude parameter. After lengthy but straightforward calculations, and rescalings, we arrive at the system of coupled equations for the wave amplitudes $A, B, C$ and $D$ :

$$
\begin{aligned}
& \mathrm{i}\left(A_{T}+v_{g 1} A_{X}\right)+\mu_{1}\left(|A|^{2}+2|B|^{2}\right) A+\mu_{2}\left(|C|^{2}+|D|^{2}\right) A=-\frac{1}{2} \varepsilon^{2} \omega_{1}^{\prime \prime} A_{X X}, \\
& \mathrm{i}\left(B_{T}-v_{g 1} B_{X}\right)-\mu_{1}\left(|B|^{2}+2|A|^{2}\right) B-\mu_{2}\left(|C|^{2}+|D|^{2}\right) B=\frac{1}{2} \varepsilon^{2} \omega_{1}^{\prime \prime} B_{X X}, \\
& \mathrm{i}\left(C_{T}+v_{g 2} C_{X}\right)+\mu_{3}\left(|C|^{2}+2|D|^{2}\right) C+\mu_{4}\left(|A|^{2}+|B|^{2}\right) C=-\frac{1}{2} \varepsilon^{2} \omega_{2}^{\prime \prime} C_{X X}, \\
& \mathrm{i}\left(D_{T}-v_{g 2} D_{X}\right)-\mu_{3}\left(|D|^{2}+2|C|^{2}\right) D-\mu_{4}\left(|A|^{2}+|B|^{2}\right) D=\frac{1}{2} \varepsilon^{2} \omega_{2}^{\prime \prime} D_{X X},
\end{aligned}
$$

where

$$
\begin{aligned}
& v_{g i}=\frac{k}{\omega_{i}} \frac{1+\alpha_{i}^{2} \delta^{2} c^{2}}{1+\alpha_{i}^{2} \delta^{2}}, \quad \omega_{i}^{\prime \prime}=\frac{1-v_{g i}^{2}+\alpha_{i}^{2} \delta^{2}\left(c^{2}-v_{g i}^{2}\right)+4 \omega_{i}^{2} \alpha_{i}\left(v_{g i}-\left(k / \omega_{i}\right)\right)\left(v_{g i}-c^{2}\left(k / \omega_{i}\right)\right)}{\omega_{i}\left(1+\alpha_{i}^{2} \delta^{2}\right)}, \\
& \mu_{1}=\frac{\delta^{2}\left(1-\alpha_{1}\right)^{4}}{4 \omega_{1}\left(1+\alpha_{1}^{2} \delta^{2}\right)}, \quad \mu_{2}=\frac{\delta^{2}\left(1-\alpha_{1}\right)^{2}\left(1-\alpha_{2}\right)^{2}}{2 \omega_{1}\left(1+\alpha_{1}^{2} \delta^{2}\right)}, \quad \mu_{3}=\frac{\delta^{2}\left(1-\alpha_{2}\right)^{4}}{4 \omega_{2}\left(1+\alpha_{2}^{2} \delta^{2}\right)}, \\
& \mu_{4}=\frac{\delta^{2}\left(1-\alpha_{1}\right)^{2}\left(1-\alpha_{2}\right)^{2}}{2 \omega_{2}\left(1+\alpha_{2}^{2} \delta^{2}\right)} .
\end{aligned}
$$


The dispersive terms in the right-hand side of (21) are small and beyond the leading order of the asymptotic multi-scale expansion method. Similar coupled NLS equations were recently derived in $[26,26 \mathrm{a}]$ for two counterpropagating waves. A system of coupled two-dimensional NLS equations for two counter-propagating waves was derived in [27]. We notice that the coupled equations (21) are valid in the general non-resonant case, when $v_{g 1} \neq v_{g 2}$ (see, for example, [28]).

The coupled NLS equations (21) have the $x$-independent coupled-wave solution:

$$
A=A_{0} \exp \left(\mathrm{i} \omega_{a} T\right), \quad B=B_{0} \exp \left(-\mathrm{i} \omega_{b} T\right), \quad C=C_{0} \exp \left(\mathrm{i} \omega_{c} T\right), \quad D=D_{0} \exp \left(-\mathrm{i} \omega_{d} T\right),
$$

where

$$
\begin{array}{ll}
\omega_{a}=\mu_{1}\left(\left|A_{0}\right|^{2}+2\left|B_{0}\right|^{2}\right)+\mu_{2}\left(\left|C_{0}\right|^{2}+\left|D_{0}\right|^{2}\right), & \omega_{b}=\mu_{1}\left(2\left|A_{0}\right|^{2}+\left|B_{0}\right|^{2}\right)+\mu_{2}\left(\left|C_{0}\right|^{2}+\left|D_{0}\right|^{2}\right), \\
\omega_{c}=\mu_{3}\left(\left|C_{0}\right|^{2}+2\left|D_{0}\right|^{2}\right)+\mu_{4}\left(\left|A_{0}\right|^{2}+\left|B_{0}\right|^{2}\right), & \omega_{d}=\mu_{3}\left(2\left|C_{0}\right|^{2}+\left|D_{0}\right|^{2}\right)+\mu_{4}\left(\left|A_{0}\right|^{2}+\left|B_{0}\right|^{2}\right) .
\end{array}
$$

Substituting (22) into (20) we obtain the solution in the form of two pairs of counter-propagating waves:

$$
\begin{aligned}
\left(\begin{array}{c}
u \\
w
\end{array}\right)= & \varepsilon\left(A_{0} \mathrm{e}^{\mathrm{i}\left(k x-\omega_{1} t+\omega_{a} \varepsilon^{2} T\right)}+B_{0} \mathrm{e}^{\mathrm{i}\left(k x+\omega_{1} t-\omega_{b} \varepsilon^{2} T\right)}\right)\left(\begin{array}{c}
1 \\
\alpha_{1}
\end{array}\right) \\
& +\varepsilon\left(C_{0} \mathrm{e}^{\mathrm{i}\left(k x-\omega_{2} t+\omega_{c} \varepsilon^{2} T\right)}+D_{0} \mathrm{e}^{\mathrm{i}\left(k x+\omega_{2} t-\omega_{d} \varepsilon^{2} T\right)}\right)\left(\begin{array}{c}
1 \\
\alpha_{2}
\end{array}\right)+\text { c.c. }
\end{aligned}
$$

Solution (23) describes a weakly nonlinear energy exchange between the two components. The only change compared to the linear solutions discussed in Section 2 is the nonlinear corrections to the frequencies $\omega_{1}, \omega_{2}$ of the linear waves, where $\omega_{1}(k)$ and $\omega_{2}(k)$ are given by (6). For $\varepsilon \rightarrow 0$, solution (23) reduces to the linear solution (8) in the case of two waves with

$$
\varepsilon A_{0}=\frac{\left|\alpha_{2}\right| A}{2\left(\alpha_{1}+\left|\alpha_{2}\right|\right)}, \quad \varepsilon C_{0}=\frac{\alpha_{1} A}{2\left(\alpha_{1}+\left|\alpha_{2}\right|\right)}, \quad B_{0}=D_{0}=0
$$

and to the linear solution (12) in the case of four waves:

$$
\varepsilon A_{0}=\varepsilon B_{0}=\frac{\left|\alpha_{2}\right| A}{2\left(\alpha_{1}+\left|\alpha_{2}\right|\right)}, \quad \varepsilon C_{0}=\varepsilon D_{0}=\frac{\alpha_{1} A}{2\left(\alpha_{1}+\left|\alpha_{2}\right|\right)} .
$$

For $\varepsilon \neq 0$, solution (23) generalizes the linear solutions by nonlinearity-induced correction of order $\mathrm{O}\left(\varepsilon^{2}\right)$ to the frequencies $\omega_{1,2}(k)$. For $c=1$, the exact nonlinear solution (18) reduces to the weakly nonlinear solution (23) in the asymptotic expansion as $\kappa \rightarrow 0$. Using the well-known approximation (e.g., [24])

$$
\operatorname{sn}(x, \kappa)=\sin x-\frac{1}{4} \kappa^{2}(x-\sin x \cos x) \cos x+\mathrm{O}\left(\kappa^{4}\right),
$$

we eliminate the secular terms by renormalizing the frequencies $\omega_{1,2}(k)$ in the solution (18). As a result, we find the asymptotic expansion with the leading order terms:

$$
\begin{aligned}
& u=\frac{2}{1+\delta^{2}}\left[\kappa \sin \left(k x-\omega_{1} t+\Omega_{1} \kappa^{2} t+\mathrm{O}\left(\kappa^{4}\right)\right)+\delta^{2} \kappa \sin \left(k x-\omega_{2} t+\Omega_{2} \kappa^{2} t+\mathrm{O}\left(\kappa^{4}\right)\right)+\mathrm{O}\left(\kappa^{2}\right)\right], \\
& w=\frac{2}{1+\delta^{2}}\left[\kappa \sin \left(k x-\omega_{1} t+\Omega_{1} \kappa^{2} t+\mathrm{O}\left(\kappa^{4}\right)\right)-\kappa \sin \left(k x-\omega_{2} t+\Omega_{2} \kappa^{2} t+\mathrm{O}\left(\kappa^{4}\right)\right)+\mathrm{O}\left(\kappa^{2}\right)\right],
\end{aligned}
$$

where

$$
\Omega_{1}=0, \quad \Omega_{2}=\frac{1+\delta^{2}}{4 \omega_{2}} .
$$


This expansion matches the weakly nonlinear solution (23) for $c=1$ with the correspondence:

$$
\begin{aligned}
& \varepsilon A_{0}=\frac{\kappa}{1+\delta^{2}}, \quad \varepsilon C_{0}=\frac{\kappa \delta^{2}}{1+\delta^{2}}, \quad B_{0}=D_{0}=0, \quad \omega_{a}=\omega_{b}=\omega_{d}=0, \\
& \omega_{c} \varepsilon^{2}=\mu_{3}\left|C_{0}\right|^{2} \varepsilon^{2}=\Omega_{2} \kappa^{2}
\end{aligned}
$$

since for $c=1$ :

$$
\mu_{1}=\mu_{2}=\mu_{4}=0, \quad \mu_{3}=\frac{\left(1+\delta^{2}\right)^{3}}{4 \omega_{2} \delta^{4}} .
$$

\section{Conclusion}

The situation when the linear dispersion relation has two or more branches for the same wavenumber is typical in multi-component systems. Many interesting phenomena can take place in such systems, including wave resonances and gap solitons. In this paper, by considering a system of coupled Klein-Gordon equations, we have discussed the processes involving the exchange of energy between the physical components of the system. The energy exchange constitutes an essential feature of multi-component systems. It would be interesting to study the energy exchange processes in other physical systems admitting a similar dispersion relation. For example, an attractive candidate is the sine-Gordon-d'Alembert systems introduced in the study of the propagation of electroacoustic walls in elastic ferroelectrics (see [8,9]) and magnetoacoustic domain walls in elastic ferromagnets [10] (see also [29,30]).

Harmonic waves in a scalar sine-Gordon equation are modulationally unstable with respect to amplitude variations. Since the system (2) with $c=1$ reduces to the sine-Gordon equation (15) and to the wave equation (16), the coupled linear and nonlinear waves are expected to be modulationally unstable too. In the general case, stability of coupled-wave solutions can be considered in the framework of coupled NLS equations (21) with the small dispersive terms in the right-hand side of (21) (see [26,26a,27] and references there in). Analytical and numerical results on stability of coupled-wave solutions will be published elsewhere (see [31] for the influence of modulational instability on the two-wave solution).

\section{Acknowledgements}

We thank V.A. Baikov, R.H.J. Grimshaw, C. Martel, and A.V. Mikhailov for useful discussions. We are also thankful to the referee for useful comments and references. We appreciate support and hospitality of the Department of Mathematical Sciences of Loughborough University. Research visit of DEP was supported by EPSRC grant \#GR/R88885/01.

\section{References}

[1] I.Sh. Akhatov, V.A. Baikov, K.R. Khusnutdinova, Non-linear dynamics of coupled chains of particles, J. Appl. Math. Mech. 59 (1995) 353-361.

[1a] K.R. Khusnutdinova, V.V. Silberschmidt, Lattice modelling of nonlinear waves in a bi-layer with delamination, Proc. Estonian Acad. Sci. Phys. Math. 52 (2003) N1, 63-75.

[2] L.I. Slepyan, Some basic aspects of crack dynamics, in: G.P. Cherepanov (Ed.), Fracture. A Topical Encyclopedia of Current Knowledge, Krieger Publishing Co., Malabar, FL, 1998.

[3] V.Ya. Antonchenko, A.S. Davydov, A.V. Zolotaryuk, Solitons and proton motion in ice-like structures, Phys. Stat. Sol. (b) 115 (1983) 631-640.

[4] A.V. Zolotaryuk, K.H. Spatschek, E.W. Ladke, Stability of activation-barrier-lowering solitons, Phys. Lett. A 101 (1984) 517-520.

[5] D. Hochstrasser, H. Büttner, H. Desfontaines, M. Peyrard, Solitons in hydrogen-bonded chains, Phys. Rev. A 36 (1988) $5332-5341$. 
[6] St. Pnevmatikos, Soliton dynamics of hydrogen-bonded networks: a mechanism for proton conductivity, Phys. Rev. Lett. 60 (1988) 1534-1537.

[7] M. Remoissenet, Nonlinear excitations in a compressible chain of dipoles, J. Phys. C 14 (1981) L335-L338.

[8] J. Pouget, G.A. Maugin, Solitons and electroacoustic interactions in ferroelectric crystals. I. Single solitons and domain walls, Phys. Rev. B 30 (1984) 5306-5325.

[9] J. Pouget, G.A. Maugin, Solitons and electroacoustic interactions in ferroelectric crystals. II. Interaction of solitons and radiations, Phys. Rev. B 31 (1985) 4633-4649.

[10] G.A. Maugin, A. Miled, Solitary waves in elastic ferromagnets, Phys. Rev. B 33 (1986) 4830-4842.

[11] M.K. Sayadi, J. Pouget, Soliton dynamics in a microstructured lattice model, J. Phys. A 24 (1991) 2151-2172.

[12] K.R. Khusnutdinova, Non-linear waves in a double row particle system, Vestnik MGU, Math. Mech. 2 (1992) 71-76.

[13] S. Yomosa, Soliton excitations in deoxyribonucleic acid (DNA) double helices, Phys. Rev. A 27 (1983) 2120-2125.

[14] L.V. Yakushevich, Nonlinear Physics of DNA, Wiley, Chichester, 1998.

[15] A.V. Zhiber, N.H. Ibragimov, A.B. Shabat, Equations of Liouville type, Dokl. Akad. Nauk SSSR 249 (1979) 26-29.

[16] S. Lie, Discussion der Differentialgleichung $\partial^{2} z / \partial x \partial y=F(z)$, Arch. für Math. og Naturv. 6 (1881) 112-124.

[17] N.H. Ibragimov (Ed.), CRC Handbook of Lie Group Analysis of Differential Equations, vol. 1, Symmetries, Exact Solutions and Conservation Laws, CRC Press, Boca Raton, FL, 1994.

[18] A.V. Zhiber, A.B. Shabat, Klein-Gordon equations with a nontrivial group, Dokl. Akad. Nauk SSSR 247 (1979) $1103-1107$.

[19] T.A. Kontorova, Ya.I. Frenkel, On the theory of plastic deformation and twinning I, II, Zh. Eksp. Teor. Fiz. 8 (1938) 89-95, $1340-1368$.

[20] O.M. Braun, Yu.S. Kivshar, Nonlinear dynamics of the Frenkel-Kontorova model, Phys. Rep. 306 (1998) 1-108.

[21] L.I. Mandelshtam, Lectures on the Theory of Oscillations, Nauka, Moscow, 1972.

[22] V.I. Arnold, Mathematical Methods of Classical Mechanics, Springer, New York, 1989.

[23] E. Kamke, Differentialgleichungen. Lösungsmethoden und Lösungen. Teil 1, Akademics Verlag, Greest \& Portig, Leipzig, 1967.

[24] M. Abramowitz, I.A. Stegun, Handbook of Mathematical Functions, Dover, New York, 1970.

[25] A. Jeffrey, T. Kawahara, Asymptotic Methods in Nonlinear Wave Theory, Pitman, London, 1982.

[26] C. Martel, E. Knobloch, J.M. Vega, Dynamics of counter propagating waves in parametrically forced systems, Physica D 137 (2000) 94-123.

[26a] C. Martel, J.M. Vega, E. Knobloch, Dynamics of counter propagating waves in parametrically driver systems: dispersion vs advection, Physica D 174 (2003) 198-217.

[27] B. Collet, J. Pouget, Modulational instability of two counter-propagating waves in a thin plate resting on elastic foundation, Proc. Estonian Acad. Sci. Phys. Math. 46 (1/2) (1997) 139-148.

[28] A.D. Craik, Wave Interactions and Fluid Flows, Cambridge University Press, Cambridge, 1985.

[29] G.A. Maugin, J. Pouget, R. Drouot, B. Collet, Nonlinear Electromechanical Couplings, Wiley, Chichester, 1992.

[30] G.A. Maugin, Nonlinear Waves in Elastic Crystals, Oxford University Press, Oxford, 1999.

[31] S.D. Griffiths, R.H.J. Grimshaw, K.R. Khusnutdinova, The influence of modulational in stability on energy exchange in coupled Sine-Gordon equations, Theor. and Math. Physics (to appears). 